

\title{
CHALMERS
}

UNIVERSITY OF TECHNOLOGY

\section{Waveguide-to-substrate transition based on unilateral substrateless finline structure: Design, fabrication, and characterization}

Downloaded from: https://research.chalmers.se, 2023-04-26 15:10 UTC

Citation for the original published paper (version of record):

López, C., Desmaris, V., Meledin, D. et al (2020). Waveguide-to-substrate transition based on unilateral substrateless finline structure: Design,

fabrication, and characterization. IEEE Transactions on Terahertz Science and Technology, 10(6): 668-676. http://dx.doi.org/10.1109/TTHZ.2020.3020683

N.B. When citing this work, cite the original published paper.

(O2020 IEEE. Personal use of this material is permitted.

However, permission to reprint/republish this material for advertising or promotional purposes 


\title{
Waveguide to Substrate Transition Based on Unilateral Substrateless Finline Structure: Design, Fabrication, and Characterization
}

\author{
C. López, V. Desmaris, D. Meledin, A. Pavolotsky and V. Belitsky, Senior Member, IEEE
}

\begin{abstract}
We report on a novel waveguide-to-substrate transition with prospective use for broadband mixer design. The transition employs a substrateless finline, i.e., a unilateral finline structure with the substrate removed between the fins. This distinctive feature diminishes the overall insertion loss and facilitates matching with the waveguide. The transition is designed on a thin silicon substrate covered by a superconducting niobium thin layer. An auxiliary Au layer situated on top of the Nb layer provides grounding for the fins and facilitates the mounting process in the split-block waveguide mount. Aiming to compare simulations with measurements, a back-to-back transition arrangement for the 211-373 GHz frequency band was designed, fabricated, and characterized at cryogenic temperatures. The simulation results for the back-to-back structure show an insertion loss of less than $0.6 \mathrm{~dB}$ in the whole band, i.e., $0.3 \mathrm{~dB}$ per transition. Furthermore, a remarkable fractional bandwidth of $55 \%$ with a return loss better than $15 \mathrm{~dB}$ is predicted. Experimental verification shows consistent results with simulations.
\end{abstract}

Index Terms - Substrateless Finline, Broadband Waveguide to Substrate Transition, Superconducting Transition.

\section{INTRODUCTION}

$\mathrm{S}$ INCE THz receivers rely on thin-film technology for mixer fabrication, waveguide to substrate transitions have a crucial role in the performance of the system. A proper impedance matching together with low losses are both essential features required in the design of such transitions. Moreover, the mixer chip mounting tolerance is also of high importance.

Due to its broad fractional bandwidth and ease of fabrication, E-probes have been widely adopted in the $\mathrm{THz}$ receivers field, e.g., [1],[2]. In these transitions, the impedance matching strongly depends on the position of the probe with respect to a waveguide backshort [3],[4]. Thus, the mounting accuracy and machining tolerances are critical for the performance, especially at mm-wave and $\mathrm{THz}$ frequencies. Other less conventional approaches have been investigated over the years. In [5], a dipole is employed to transfer energy from a rectangular waveguide into conductor backed-coplanar waveguide in the $340-380 \mathrm{GHz}$ frequency range. Meanwhile, in [6], a planar dual-dipole antenna is suggested as a transition with a potential fractional bandwidth of $31.8 \%$. However, none of these structures can address all the outlined requirements simultaneously, i.e., a broadband response with proper impedance matching and low losses without compromising mounting tolerance.

As an alternative solution, unilateral finline structures [7] are more tolerant in terms of the accuracy in their waveguide positioning. Furthermore, they are simply grounded to the waveguide through beamleads. In [8], a $\mathrm{THz}$ receiver employing a finline mixer is demonstrated. Nevertheless, this approach results in problems related to impedance matching between the waveguide and the substrate, which degrades its performance and prevents its application over large operational bandwidths. Moreover, the concentration of the field in the fingap results in large conductor and dielectric losses due to the presence of the dielectric slab [9]. Therefore, if the substrate confined by the fins is removed, it will theoretically alleviate both problems, i.e., reduce dielectric losses and improve matching.

\section{THEORY AND DESIGN}

In the novel design proposed in this paper, we suggest realizing the impedance matching between a full-height waveguide and a slotline in two stages. The first stage employs a unilateral finline structure in which the substrate has been etched away [10], from now on referred to as "substrateless finline". Meanwhile, a 2-section slotline Chebyshev transformer is implemented to reach the desired slotline impedance of $70 \mathrm{Ohm}$, considered suitable for further coupling to a prospective mixer. In Fig. 1, the proposed transition is depicted. For verification and demonstration purposes, the transition is intended for cryogenic operation in the frequency band 211-375 GHz. Hence, the waveguide dimensions were selected to cover the design band adequately, i.e., 800x400 $\mu \mathrm{m}$. The transition has been designed on a $30 \mu \mathrm{m}$ silicon substrate covered by a layer of $400 \mathrm{~nm}$ thick superconducting niobium. The fins gradually converge into a slotline defined by the niobium layer. A $5 \mu \mathrm{m}$ thick Au layer deposited over the niobium film extends $100 \mu \mathrm{m}$ beyond the substrate in each

The authors are with the Group for Advanced Receiver Development (GARD), Department of Space, Earth and Environment, Chalmers University of Technology, Gothenburg, Sweden 


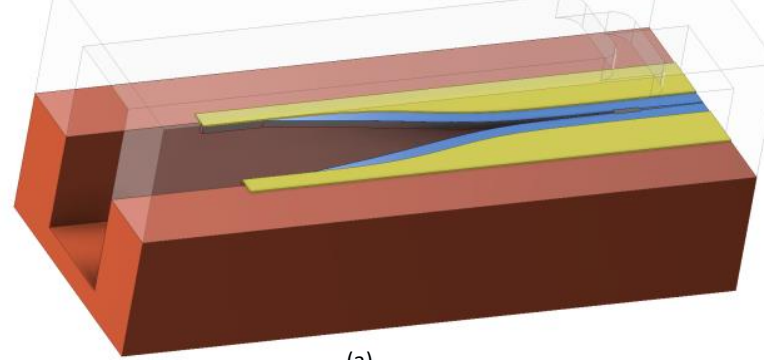

(a)

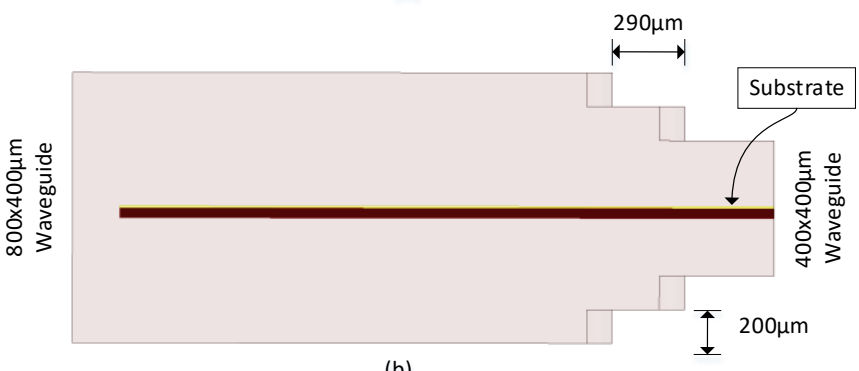

(b)
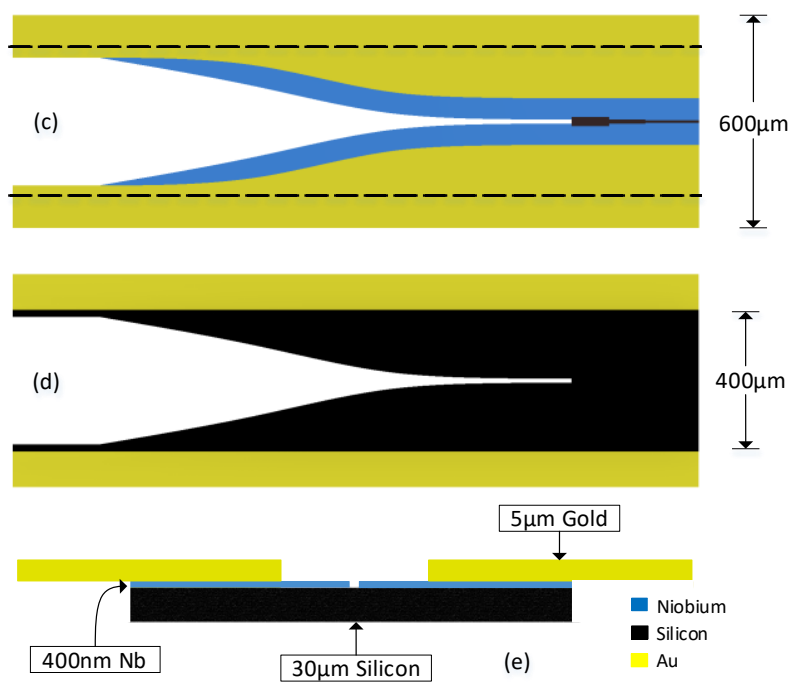

Fig. 1. Proposed waveguide to substrate transition. (a) Transition mounted in the block. Beamleads are clamped by the two halves of the split block to provide grounding. (b) Lateral view of the structure mounted in the waveguide. The input $800 \times 400 \mu \mathrm{m}$ waveguide is reduced to $400 \times 400 \mu \mathrm{m}$ in 2 steps to prevent the propagation of unwanted waveguide modes. (c) Top view of the transition. Au beamleads extend by $100 \mu \mathrm{m}$ from each side of the silicon substrate. The dashed lines illustrate the waveguide dimension. (d) The bottom view reveals the profile of the silicon substrate. (e) Transition viewed from slotline side. The different material thicknesses are appreciated. For the sake of clarity, this image is shown not to scale.

direction and serves as beamleads. Thus, upon closing the waveguide split block, the Au layer gets clamped and crashed between the two waveguide halves, providing grounding for the fins. Furthermore, the waveguide width is gradually reduced from $800 \mu \mathrm{m}$ to $400 \mu \mathrm{m}$ in 2 steps to obtain subcritical dimensions $400 \times 400 \mu \mathrm{m}$. These steps are introduced further in the transition when the field has already concentrated between the fins. As a result, the waveguide shrinking has almost no influence over the impedance of the transition but provides good isolation against unwanted waveguide modes.

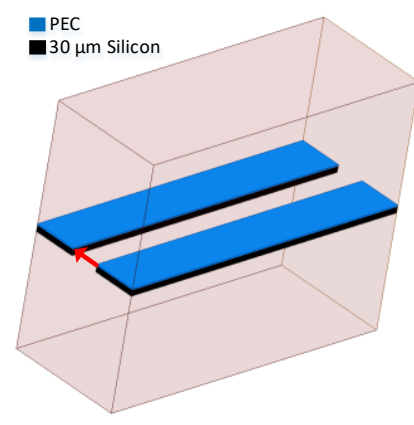

(a)

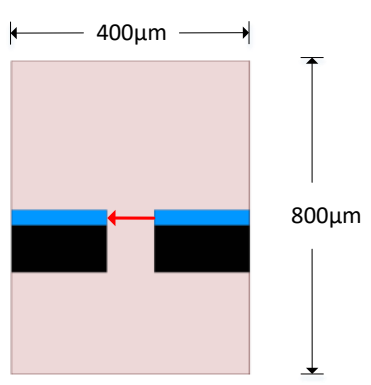

(b)
Impedance vs Width

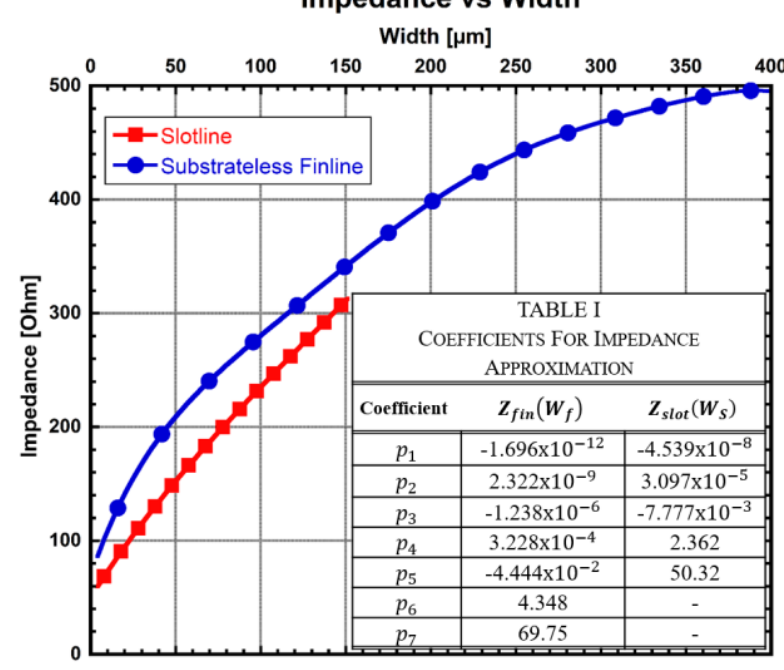

(c)

Guided Lambda vs Width

Width $(\mu \mathrm{m})$

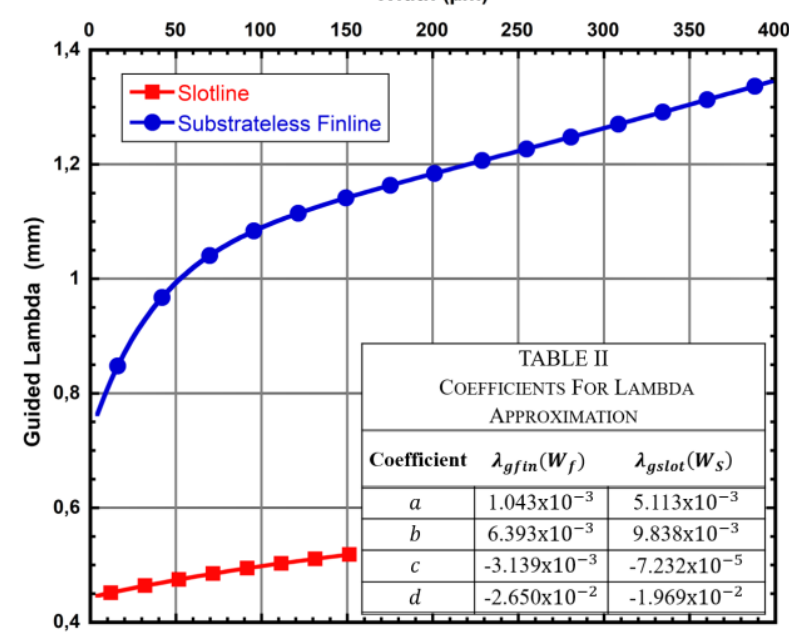

(d)

Fig. 2. Approximations for the characteristic impedance and the guided wavelength of substrateless finlines and superconducting slotlines. Note they are valid for a $30 \mu \mathrm{m}$ silicon substrate and within the frequency range 211-375 GHz. In addition, the slotline approximations are only applicable for slot-width between 4 and $150 \mu \mathrm{m}$. (a) Simulated subtrateless finline section. This structure was simulated with different fin-gaps to obtain the data for the polynomic approximations. A similar structure was employed for the slotline approximation. The red arrow depicts the electric field excitation of the fin-gap. (b) Front view of substratless finline section. The finline section was located inside a $800 \times 400 \mu \mathrm{m}$ waveguide. This image is shown not to scale. (c) Polynomial approximations for the characteristic impedance as a function of slot width. The polynomial coefficients can be found in the insert (Table I). (d) Exponential approximation for guided wavelength vs. width. The coefficients are depicted in the insert (Table II). 
(a)

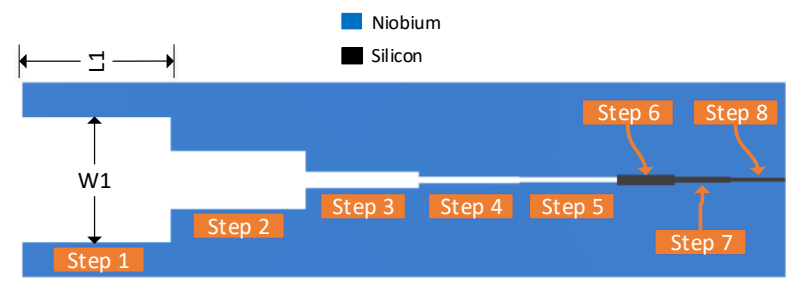

(b)

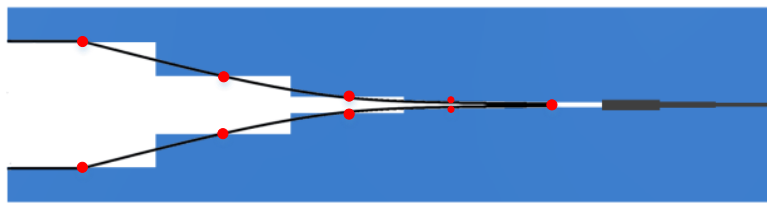

Fig. 3. Chebyshev transformers in substrateless finline and superconducting slotline. (a) The complete impedance transformation involves eight steps. The first five steps correspond to the substrateless finline transformer. Meanwhile, steps 6 to 8 are part of the superconducting slotline transformer. (b) The spline is drawn through the center of steps 1 to 5 . This improves the response at the higher side of the band.

TABLE III

CHEBYSHEV STEP TRANSFORMERS

\begin{tabular}{|c|c|c|c|c|}
\hline & Step & $\begin{array}{c}\text { Approximated } \\
\text { Impedance } \\
\text { [Ohm] }\end{array}$ & $\begin{array}{l}\text { Width } \\
{[\mu \mathrm{m}]}\end{array}$ & Length $[\mu \mathrm{m}]$ \\
\hline \multirow{5}{*}{$\begin{array}{c}1^{\text {st }} \text { Step } \\
\text { Transformer }\end{array}$} &  & ב445 & 256.8 & 307 \\
\hline & 2 & 304 & 119 & 278 \\
\hline & 3 & 177 & 33.92 & 234 \\
\hline & 4 & 121 & 13.52 & 208 \\
\hline & 5 & 108 & 9.76 & 201 \\
\hline \multirow{2}{*}{$\begin{array}{c}2^{\text {nd }} \text { Step } \\
\text { Transformer }\end{array}$} & 6 & 98.88 & 21.97 & 118.9 \\
\hline & 7 & 78.84 & 12.5 & 115.3 \\
\hline $\begin{array}{c}\text { Slotline } \\
\text { Load }\end{array}$ & 8 & 70 & 8.5 & 113.6 \\
\hline
\end{tabular}

\section{A. Theory of Design}

Although a large number of different methods for unilateral finline design have been reported, e.g., [11],[12], none have been formulated for substrateless finlines. Therefore, we suggest an alternative approach to define the finline profile. Employing the full-wave 3D simulator Ansys HFSS, the characteristic impedance and guided wavelength for finline sections of different widths were investigated and mapped. Hence, the impedance as a function of the fin-gap was approximated by a $6^{\text {th }}$ order polynomial. Meanwhile, the guided lambda was better described by a superposition of exponential functions,

$$
\begin{gathered}
Z_{f i n}\left(W_{f}\right)=p_{1} W_{f}^{6}+p_{2} W_{f}^{5}+p_{3} W_{f}^{4}+p_{4} W_{f}^{3} \\
+p_{5} W_{f}^{2}+p_{6} W_{f}+p_{7} \\
\lambda_{g f i n}\left(W_{f}\right)=a^{\left(b W_{f}\right)}+c^{\left(d W_{f}\right)} \\
4 \leq W_{f} \leq 400
\end{gathered}
$$

where $Z_{\text {fin }}$ and $\lambda_{\text {gfin }}$ are the characteristic impedance and guided lambda of substrateless finlines. The fin-gap is $W_{f}$. It important to note that $W_{f}$ is expressed in $\mu \mathrm{m}$.

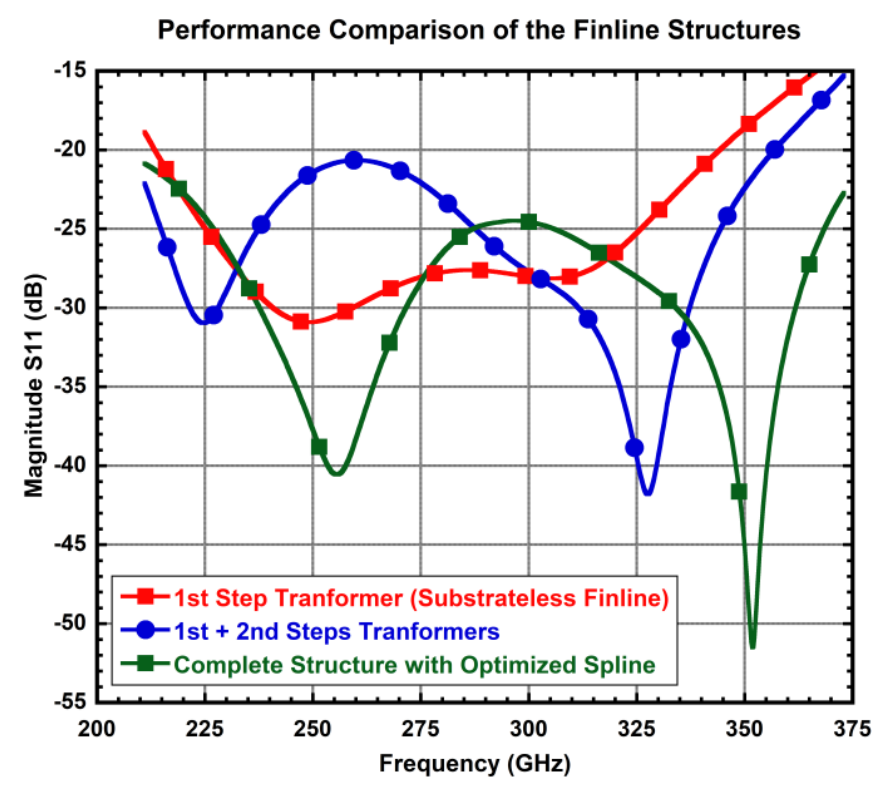

Fig. 4. Simulated performance for the standalone first step transformer and together with the second transformer. In addition, the simulated S11 magnitude for the optimized structure with spline is plotted.

TABLE IV

OPTIMIZED DESIGN WITH SPLINE

\begin{tabular}{c|c|c}
\hline \hline \multicolumn{1}{c}{ Step } & Width $[\mu \mathrm{m}]$ & Length $[\mu \mathrm{m}]$ \\
\hline \hline 1 & 360 & 490 \\
\hline 2 & 190 & 432 \\
\hline 3 & 48 & 286 \\
\hline 4 & 15 & 252 \\
\hline 5 & 11 & 120 \\
\hline 6 & 25 & 105 \\
\hline 7 & 11 & 103 \\
\hline 8 & 7 & 152 \\
\hline \hline
\end{tabular}

The same procedure was employed to explore the characteristics of superconducting slotline with $30 \mu \mathrm{m}$ silicon substrate. The approximations for superconducting slotline are the following:

$$
\begin{gathered}
Z_{\text {slot }}\left(W_{s}\right)=p_{1} W_{s}^{4}+p_{2} W_{s}^{3}+p_{3} W_{s}^{2}+p_{4} W_{s}+p_{5} \\
\lambda_{g s l o t}\left(W_{s}\right)=a^{\left(b W_{s}\right)}+c^{\left(d W_{s}\right)} \\
4 \leq W_{s} \leq 150
\end{gathered}
$$

where $Z_{\text {slot }}$ and $\lambda_{\text {gslot }}$ are the characteristic impedance and guided wavelength of a superconducting slotline, respectively and the slot-width $\left(W_{S}\right)$ is expressed in $\mu \mathrm{m}$. The impedance and wavelength functions are plotted in Fig. 2. The coefficients for the polynomial and exponential approximations can be found in the inserts to Fig. 2 (Table I and Table II, respectively).

A 5-step Chebyshev transformer was designed employing the proposed approximations. It transforms the nearly $500 \mathrm{Ohm}$ impedance of a full-height waveguide to $108 \mathrm{Ohm}$ substrateless finline, where it can be coupled to a slotline. The subsequent 2step Chebyshev slotline transformer further reduces the 




(a)

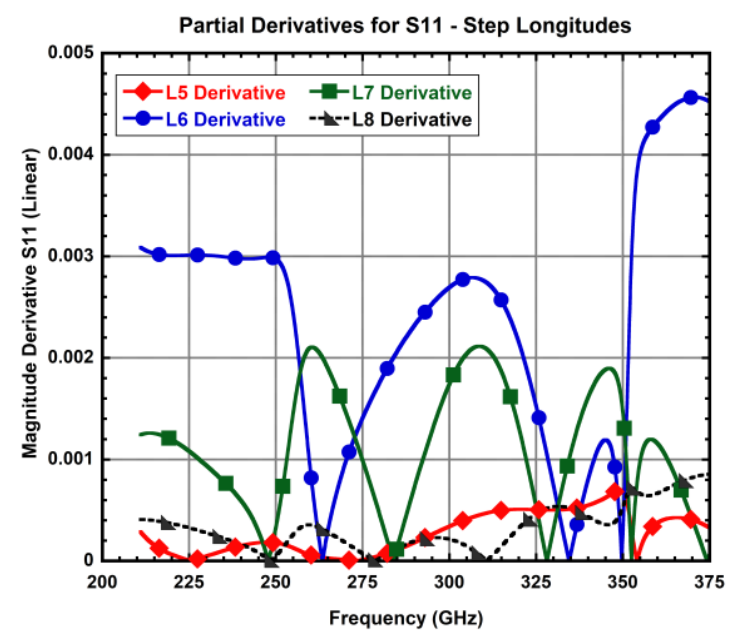

(b)

Fig. 5. Partial derivatives of S11 magnitude vs. frequency. The comparison of the derivatives reveals the sensitivity of the structure to the different parameters. (a) Partial derivatives for S11 magnitude regarding the different step widths. (b) Partial derivatives for S11 magnitude with respect to the step length.

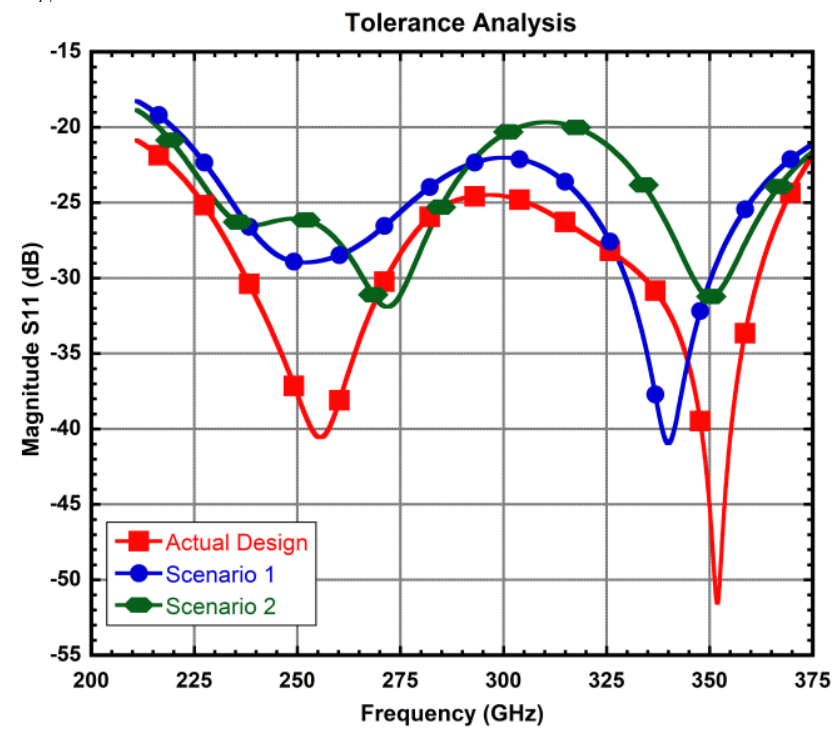

Fig. 6. Tolerance analysis for two worst-case scenarios. The first scenario introduces $+1 \mu \mathrm{m}$ in the most sensitive widths and $+5 \mu \mathrm{m}$ in the longitudes. As for the scenario 2, the widths and longitudes are decreased by the same amount. For clarity, the actual design performance is plotted in the same graph.

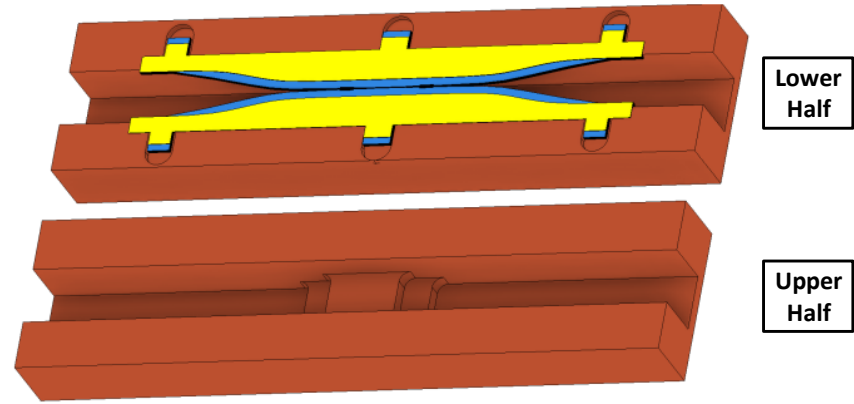

Fig. 7. Back to back arrangement. The upper and lower halves of the block are depicted separately. The structure incorporates lateral silicon tips to facilitate mounting.

impedance to $70 \mathrm{Ohm}$. The full structure can be seen in Fig. 3 . Table III provides the values for the widths and lengths of each step.

With the purpose of improving the matching of the structure, a spline curve was drawn through the center of each step. Furthermore, in Fig. 1(c), it is seen how the Au beam leads are added following a similar spline but offseted from the $\mathrm{Nb}$ spline edge. This offset is included to avoid losses on the Au layer without sacrificing good grounding.

It is important to note that the spline curve is fully defined with the width and length of the steps through which the spline line is driven. Therefore, it is useful to continue analyzing the structure using these parameters. In Table IV, the values of the parameters for the final design are shown. These values were obtained through genetic optimization routine in Ansys HFSS. Simulations of the optimized design shown a return loss better than $20 \mathrm{~dB}$ over the whole band, as it is depicted in Fig. 4.

\section{B. Tolerance Analysis}

In order to address the fabrication tolerances, different scenarios were simulated. Taking into consideration that the transition is modeled by more than 20 variables, it would require a substantial computational effort to analyze all possible variations. Therefore, a different approach was employed to identify the most sensitive variables. The $\mathrm{S}$ parameters and its partial derivatives against a geometric design parameter $v$ can be described as a function of frequency and the different geometric variables of the model as follows:

$$
S_{n, m}\left(W_{1}, W_{2}, \ldots, W_{8}, \ldots, L_{1}, L_{2}, \ldots, L_{8}, \ldots, f\right) ; \frac{\partial S_{n, m}}{\partial v}(f)
$$

where the port numbers are $\mathrm{n}$ and $\mathrm{m}$, while $W$ and $L$ correspond to the width and length of the transformer steps, respectively. The partial derivatives with greater magnitude have a more significant impact on the device performance. Since Ansys HFSS includes a feature to calculate the partial derivatives of the design, the most sensitive variables can be easily obtained. In Fig. 5(a), the derivatives for different steps widths are plotted. It is clear that the variation of $W_{8}, W_{7}, W_{6}$ and $W_{5}$ are fundamental for transition performance. Regarding the length of each step, Fig. 5(b) shows that $L_{7}$ and $L_{6}$ are the most sensitive values. This result is expected, since the functions that approximate the characteristics of substrateless finline and 


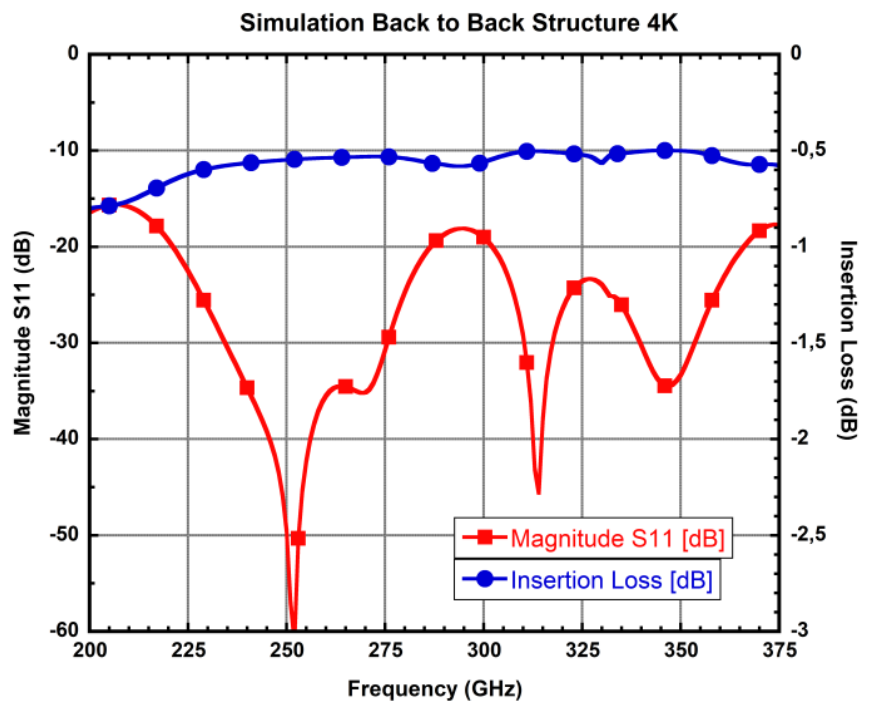

Fig. 8. Simulated scattering parameters for the back to back arrangement. The S11 magnitude is better that $15 \mathrm{~dB}$ and the insertion loss is below $0.6 \mathrm{~dB}$ in the 211-375 GHz frequency band.

slotline are more sensitive for lower values of $W$ and $L$. It is important to remark that all the parameters of the model were analyzed by the same mean. This includes material thicknesses, Au profile definition, and the positioning of structure inside the waveguide. The results showed that these parameters have considerably less influence in the design performance than $W$ and $L$.

Since the device fabrication relies on photolithography and thin-film technology, it can be assumed that any deviation on the pattern will impact all the steps simultaneously. Thus, two worst-case scenarios could be considered. The first involves increasing $1 \mu \mathrm{m}$ in the most sensitive widths and $5 \mu \mathrm{m}$ in the critical lengths. Meanwhile, in the second one, these parameters were reduced by the same amount. From the analysis of both scenarios depicted in Fig. 6, as far as the fabrication tolerances are below $1 \mu \mathrm{m}$ for the widths and $5 \mu \mathrm{m}$ for the longitudes, the overall performance will not be affected.

\section{Back to Back Transition}

For the sake of comparison between simulations with measurements, a test structure comprising two transitions in a back-to-back arrangement was designed. In order to facilitate mounting and improve the mechanical strength of the assembly, silicon tips were introduced in the structure, as it is depicted in Fig. 7. These tips extend from the primary substrate and fit inside cavities located in the lower half of the split block. The simulation results for the performance of the back-to-back structure at $4 \mathrm{~K}$ are depicted in Fig. 8. For cryogenic temperature, an insertion loss less than $0.6 \mathrm{~dB}$ and a return loss better than $15 \mathrm{~dB}$ is achieved over the whole band.

\section{FABRICATION}

The samples were fabricated in-house following the fabrication process detailed in Fig. 9. Silicon-On-Insulator technology was employed to facilitate the handling and definition of the ultrathin $30 \mu \mathrm{m}$ silicon chip during the process. The handle layer was $300 \mu \mathrm{m}$. Furthermore, the $2 \mu \mathrm{m}$ silicon

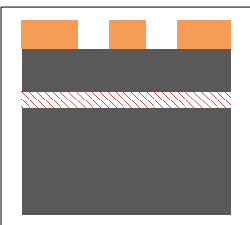

(a)
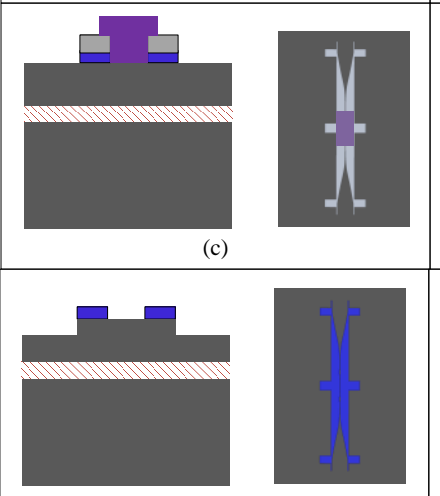

(e)

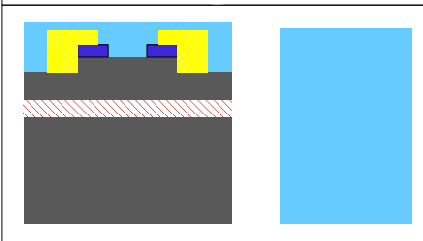

(g)

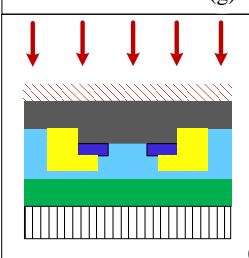

(i)

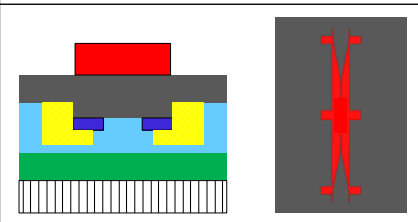

(k)

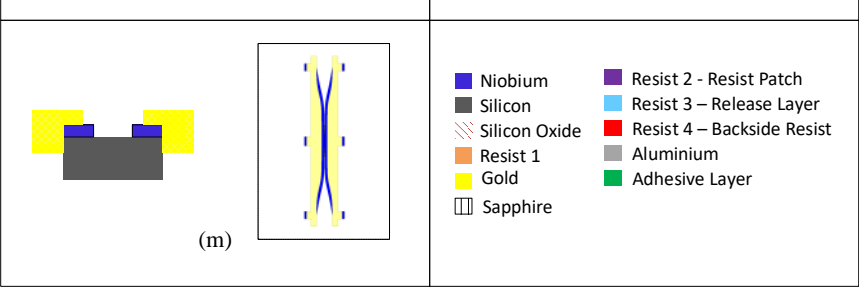

Fig. 9. Steps of the fabrication process. (a) First lithography (b) $\mathrm{Nb}$ and Aluminium deposition and lift off. (c) Protection of slotline transformer with resist (protective resist patch). (d) Definition of substrateless finline through dry etching silicon. (e) Removing of aluminum hard mask and resist patch (f) Electroplating of $\mathrm{Au}$ beamleads. For simplicity, the intermediate steps required for patterning the electroplated Au layer are not showed. (g) Spinning of release-layer. (h) The sample is mounted upside down over a transparent sapphire wafer using an adhesive layer. (i) The Si handle layer is etched away. (j) Etching of silicon oxide stop layer (k) Backside lithography. (1) Dry etch of the remaining Si device layer. (m) Release of the devices.

oxide served as a natural etch stop for the dry etch step.

The process started with spinning and baking a positive photoresist over a carefully cleaned silicon substrate. The first 

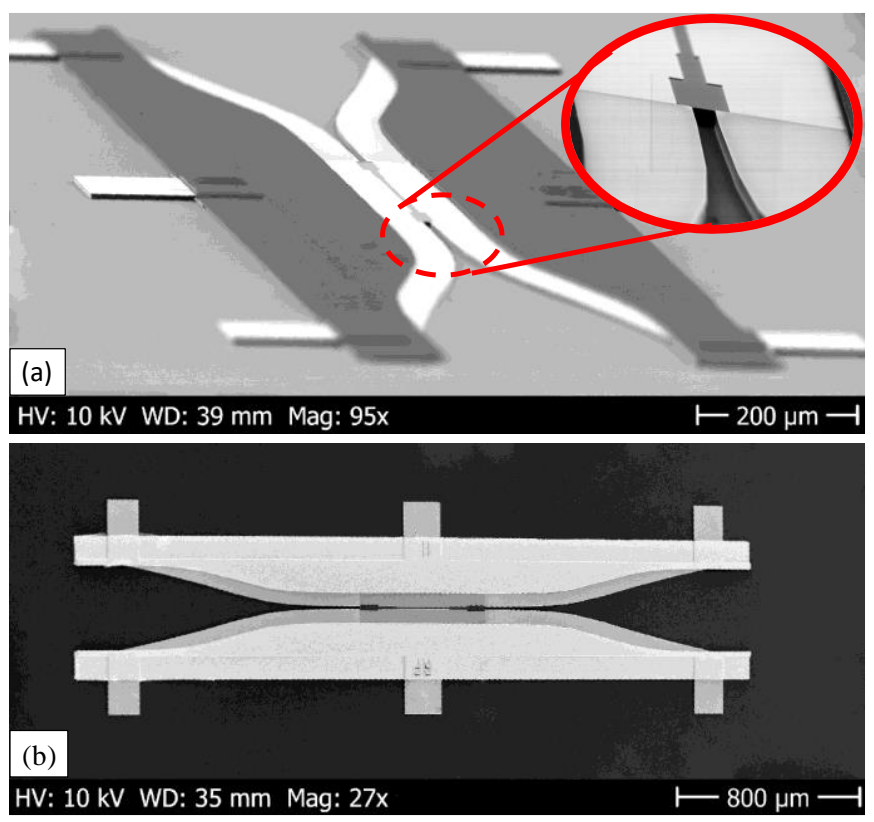

Fig. 10. Scanning Electron Microscope images of the fabricated back-to-back device. (a) Finline structure after etching the fist $5 \mu \mathrm{m}$ of the Si device layer. The central area is magnified to appreciate the verticality of the side walls. (b) Top view of the realeased device.

lithography step created the lift-off mask. Once the photoresist was developed, a thin $\mathrm{Nb}$ film with an approximate thickness of $400 \mathrm{~nm}$ was deposited by DC magnetron sputtering. Furthermore, a $100 \mathrm{~nm}$ Al film was sputtered over the $\mathrm{Nb}$ in the same vacuum run. The lift-off in acetone defined the main features of the finline structure and provided a hard mask for the subsequent anisotropic Si etching process. In a second lithography step, a resist patch was created to preserve the silicon layer that outlines the slotline. The $5 \mu \mathrm{m}$ of the unprotected silicon were anisotropically etched with the help of the Bosch process. This guarantees an accurate definition of the substrateless finline structure. Afterward, the resist patch and the $\mathrm{Al}$ hard mask were removed. The $5 \mu \mathrm{m}$ Au beamleads were created by photolithography and electroplating process. A $\mathrm{Ti} / \mathrm{Au}$ bilayer was used as an electroplating seed.

For backside processing, the chip was mounted upside down on a transparent 4-inch sapphire wafer using a release layer and adhesive layer. The next step consisted of etching the thick silicon handle layer using the buried $\mathrm{SiO}_{2}$ layer as an etch stop. The $\mathrm{SiO}_{2}$ was later stripped away by reactive ion etching in CF4 gas. The latter allows the final lithography step on top of the remaining $25 \mu \mathrm{m}$ thick silicon layer. Backside lithography over a thick photoresist provided the etch mask for the final anisotropic etching that completed the device definition. Lastly, the adhesive layer and the release layer were removed in a solvent, releasing and separating the samples. SEM pictures of the fabricated devices are depicted in Fig. 10.

\section{BACK-TO-BACK MEASUREMENTS}

The characterization of the device was done at $4 \mathrm{~K}$ in a cryostat with a closed-cycle refrigerator. The $S$ parameters were obtained with a Keysight PNA-X 5242A. The performance of the back-to-back transition was measured using three VDI
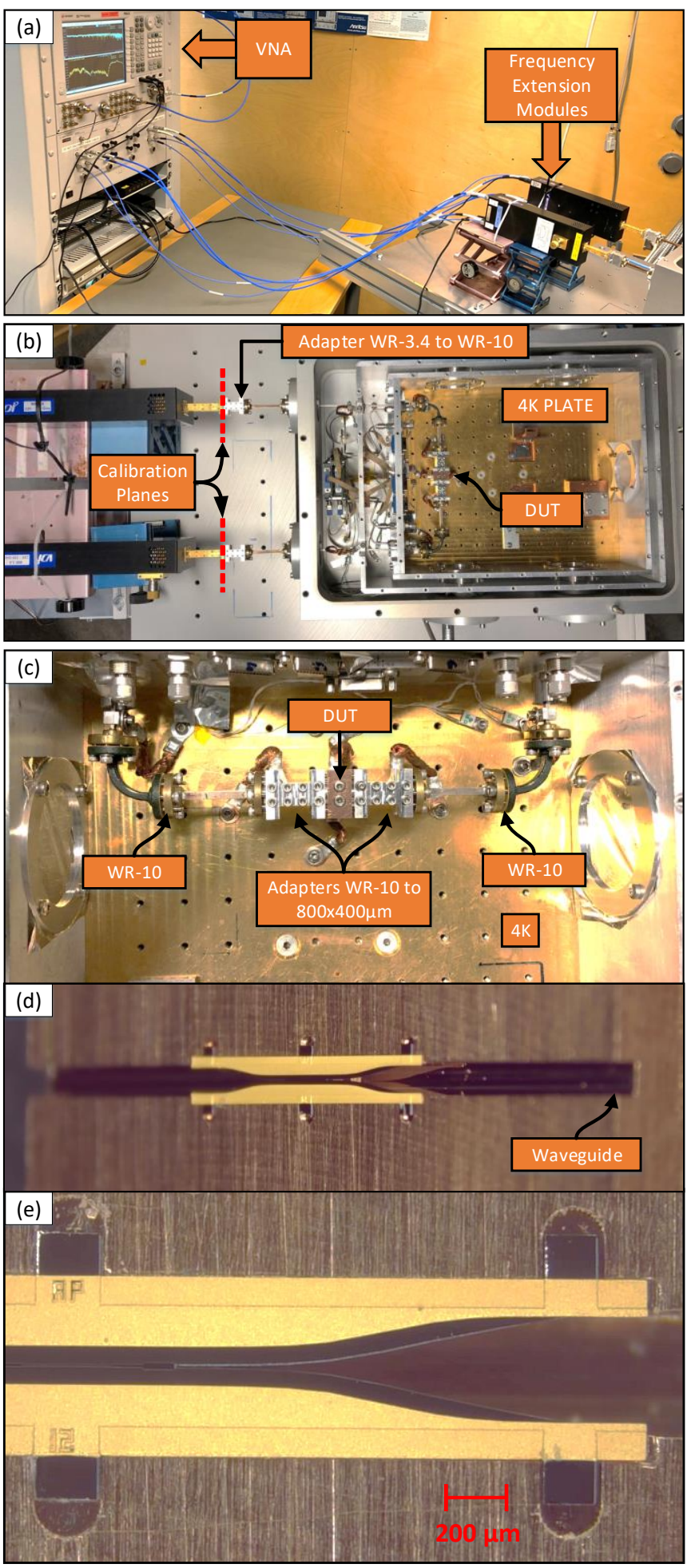

Fig. 11. Measurement setup. (a) WR-3.4 frequency extension modules and VNA (b) The extension modules are connected to the WR-10 waveguide chain using adapters. The calibration plane is located outside the cryostat. (c) Waveguide chain inside the cryostat. (d) Finline mounted in the telluriumcopper block with waveguide dimension 800x420 $\mu \mathrm{m}$ (e) Magnified image of the mounted finline.

frequency extension modules, i.e., WR-5.1, WR-3.4, and WR2.2 , to cover the whole frequency band. The measurement setup is shown in Fig. 11. It is comprised of a WR-10 symmetrical waveguide system with specially designed waveguide adapters 
Relative Measurements Back-to-Back Transition at $4 \mathrm{~K}$ and $10 \mathrm{~K}$

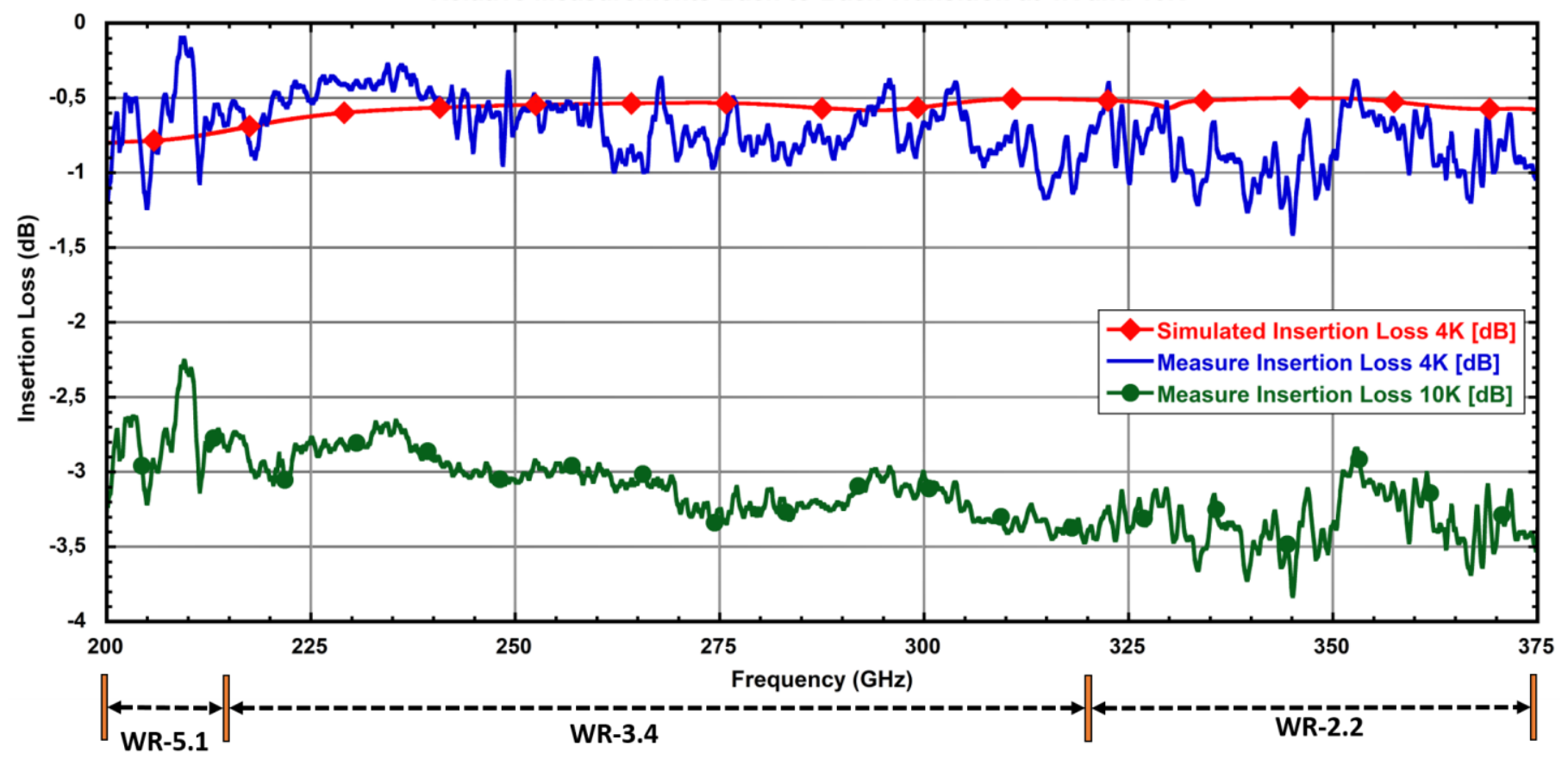

Fig. 12. Measured and simulated insertion loss for $4 \mathrm{~K}$ together with the measured frequency bands. The losses above the transition temperature for $\mathrm{Nb}$ are also plotted.

TABLE V

State Of The ARt Waveguide To Substrate TRAnsitions

\begin{tabular}{|c|c|c|c|c|c|}
\hline Reference & Transition Type & $\begin{array}{c}\text { Intended } \\
\text { Frequency Band } \\
{[\mathrm{GHz}]} \\
\end{array}$ & $\begin{array}{l}\text { Fractional BW @ } 15 \\
\text { dB Return Loss }\end{array}$ & *Insertion Loss $[\mathrm{dB}]$ & Measurement Method \\
\hline [3] & Radial Probe to microstrip & $375-500$ & $\sim 30 \%$ & 0.15 & $\begin{array}{c}\text { Scale Model Verification - } \\
\text { Warm }\end{array}$ \\
\hline [14] & Radial Probe to microstrip & $140-220$ & $44.4 \%$ & 0.45 & $\begin{array}{c}\text { Estimation from Cryogenic } \\
\text { Measurement }\end{array}$ \\
\hline [4] & Radial Probe to microstrip & $270-430$ & $45.7 \%$ & Not Reported & $\begin{array}{c}\text { Scale Model Verification - } \\
\text { Warm }\end{array}$ \\
\hline [6] & Dual-dipole antenna to microstrip & $580-800$ & $31.8 \%$ & 2 & $\begin{array}{c}\text { Scale Model Verification - } \\
\text { Warm }\end{array}$ \\
\hline [16] & Unilateral Finline to slotline & $550-750$ & $30.7 \%$ & Negligible & Simulation \\
\hline [17] & Unilateral Finline to slotline & $170-265$ & $38.2 \%$ & 0.5 & $\begin{array}{l}\text { Measure Back to Back } \\
\text { Transition - Warm }\end{array}$ \\
\hline $\begin{array}{l}\text { This } \\
\text { Work }\end{array}$ & Unilateral Substrateless Finline & 211-375 & $55 \%$ & $\sim 0.5$ & $\begin{array}{c}\text { Cryogenic Back to Back } \\
\text { Insertion Loss } \\
\text { Measurement }\end{array}$ \\
\hline
\end{tabular}

*The insertion loss is for a single transition as reported in referenced papers.

for each band of the frequency extension modules. A pair of adapters were located at the room temperature side. They transformed the WR-10 waveguide dimensions into the required size for the extension modules and vice versa. Furthermore, adapters placed at $4 \mathrm{~K}$ allow the connection of the DUT into the WR-10 system. The DUT block was fabricated in tellurium copper through direct milling. To facilitate the mounting of the device, the dimension of the waveguide width was increased by $20 \mu \mathrm{m}$, i.e., $800 \times 420 \mu \mathrm{m}$. Simulations showed this minor modification did not impact the overall performance of the device. Since $3.06 \mathrm{~mm}$ long sections of straight waveguide were added at each side of the DUT, the overall length of the block was $1 \mathrm{~cm}$. Two cooling straps were attached from the block to the $4 \mathrm{~K}$ plate to ensure proper thermal contact. Besides, two additional straps were coupled to the waveguide adapters.

The direct measurement of $\mathrm{S}$ parameters at cryogenic temperatures always poses considerable challenges, especially at high frequencies. Since the overall losses of the system change as the temperature decreases, room temperature calibrations performed at the DUT planes will dramatically drift during the cooling process. At lower frequencies, this is usually corrected with a short circuit placed at the original calibration plane. Thus, when the short circuit is cooled down, the calibration drift can be easily tracked and corrected, as described in [13]. However, this method is not feasible at higher 
frequencies since the thermal contraction in the waveguide chain is comparable to the guided wavelength. Such significant differences in electrical length preclude an accurate correction of room temperature calibrations. Therefore, an alternative approach for measuring the losses of the DUT was implemented. A standard TRL calibration was applied for the 220-330 GHz band at room temperature. The calibration planes are depicted in Fig. 11b. This calibration was used for measuring the DUT at $4 \mathrm{~K}$ and $10 \mathrm{~K}$. Afterward, a waveguide thru with the same dimensions as the DUT block was measured at the same temperatures. Since the waveguide chain was identical in both cases, the difference in the measured losses corresponds to the DUT. Thus, the insertion loss of DUT relative to a waveguide thru was obtained. The same procedure was repeated for WR-5.1 and WR-2.2 bands.

\section{RESULTS AND DISCUSSION}

The simulated and measured performance of the fabricated back to back transition is illustrated in Fig. 12. From the graph, it is clearly seen how the data obtained from different extension modules join together smoothly, confirming accurate crosscalibration during measurement with different extension modules. The back-to-back measured insertion loss at $4 \mathrm{~K}$ is less than $1 \mathrm{~dB}$ over most of the entire operational band. Meanwhile, the losses triple above the superconducting transition temperature, i.e., $9.3 \mathrm{~K}$ for $\mathrm{Nb}$. Although the experimental data was found in excellent agreement for the lower side of the band, it deviates from the predicted values at the higher end. Our analysis shows that it can be explained from fabrication defects and small differences between the waveguide chain of the DUT and the thru.

A comparison among the state of the art waveguide to substrate transitions intended for $\mathrm{THz}$ receivers is presented in Table V. From the table, it is clear that the majority of transitions are indirectly verified through scale models at lower frequencies. Although this method validates the design approach, it is not fully representative of the real transition performance. For instance, the survival of the device to a cooling cycle cannot be proved with scaled models. Furthermore, the thermal contraction of the different materials and stress generated during the cool-down process could impact on the performance. Additionally, mounting and positioning sensitivity can only be verified through direct measurement in the intended frequency band. To the authors' knowledge, in this work, we present the first direct measurement of a waveguide to substrate transition at cryogenic temperatures. It is worth noting that in [14], a radial probe for the WR-05 band was employed in a SIS frequency multiplier fabricated on a silicon substrate. The insertion loss of the probe was estimated from the cryogenic measurements of the multiplier. Nevertheless, this did not constitute a direct measurement.

By comparing the characteristics of the different transitions, it is straightforward to see that substrateless finlines stand out by their considerable fractional bandwidth. Moreover, its insertion loss is comparable with existing state-of-the-art solutions on silicon substrates.

\section{CONCLUSION}

In this paper, we have introduced a novel broadband waveguide to substrate transition, which employs a new kind of finline structure, i.e., substrateless finlines. Furthermore, we have modeled the complete transition and established a set of design guidelines. Following these guidelines, a back-to-back transition for the 211-375 GHz frequency band was designed, simulated, and fabricated. Simulation results of the back-toback arrangement showed a remarkable 55\% fractional bandwidth with better than $15 \mathrm{~dB}$ return loss over the whole band. Moreover, the predicted back-to-back insertion loss was $0.6 \mathrm{~dB}$ over most of the operational RF band. The back-to-back structure was experimentally verified at cryogenic temperatures in the $200-375 \mathrm{GHz}$ frequency range. To the best knowledge of the authors, this constitutes the first direct measurement of a waveguide to substrate transition at cryogenic temperatures. The experimental results were found in reasonably good agreement with simulations.

Due to its large bandwidth and small losses, the proposed transition can be potentially employed for broadband mixer design for radioastronomy receivers. Moreover, its mounting simplicity and straightforward design make the suggested transition hem an attractive solution for a wide variety of applications.

\section{ACKNOWLEDGMENT}

The authors would like to thank Sven-Erik Ferm and Mathias Fredrixon for the fabrication of the waveguide block and waveguide adapters.

Part of the device fabrication was done at The Electrum Laboratory, KTH Royal Institute of Technology.

The research was carried out with funding from Onsala Space Observatory.

The research leading to these results has received funding from the European Commission Seventh Framework Programme (FP/2007-2013) under grant agreement No 283393 (RadioNet3).

\section{REFERENCES}

[1] V. Belitsky, I. Lapkin, M. Fredrixon, D. Meledin, E. Sundin, B. Billade, S.-E. Ferm , A. Pavolotsky1 , H. Rashid, M. Strandberg, V. Desmaris , A. Ermakov, S. Krause, M. Olberg, P. Aghdam, S. Shafiee, P. Bergman , E. De Beck, H. Olofsson2, J. Conway, C. De Breuck, K. Immer, P. Yagoubov , F. M. Montenegro-Montes , K. Torstensson , J.-P. PérezBeaupuits, T. Klein, W. Boland, A. M. Baryshev, R. Hesper, J. Barkhof, J. Adema, M. E. Bekema, and A. Koops, "SEPIA - a new single pixel receiver at the APEX telescope," Astron. \& Astrophys., vol. 612, pp.1-12, Apr. 2018, Art. no. A23, doi: 10.1051/0004-6361/201731458.

[2] V. Belitsky, M. Bylund, V. Desmaris , A. Ermakov , S.-E. Ferm , M. Fredrixon, S. Krause, I. Lapkin, D. Meledin , A. Pavolotsky, H. Rashid , S. Shafiee, M. Strandberg, E. Sundin, P. Yadranjee Aghdam, R. Hesper , J. Barkhof, M. E. Bekema, J. Adema, R. de Haan, A. Koops, W. Boland, P. Yagoubov, G. Marconi, G. Siringo, E. Humphreys, G. H. Tan , R. Laing , L. Testi , T. Mroczkowski , W. Wild, K. S. Saini4, and E. Bryerton, "ALMA Band 5 receiver cartridge-Design, performance, and commissioning," Astron. \& Astrophys., vol. 611, pp.1-10, Mar. 2018, Art. no. A23, doi: 10.1051/0004-6361/201731883.

[3] C. Risacher, V. Vassilev, A. Pavolotsky, V. Belitsky, "Waveguide-tomicrostrip transition with integrated bias-T," IEEE Microw. Wireless 
Compon. Lett., vol 13, no. 7, pp. 262-264, Jul. 2007, doi: 10.1109/LMWC.2003.815182.

[4] J. W. Kooi, G. Chattopadhyay, S. Withington, F. Rice, J. Zmuidzinas, C. Walker, \& G. Yassin, "A full-height waveguide to thin-film microstrip transition with exceptional RF bandwidth and coupling efficiency," Int. J. of Infrared and Millimeter Waves, vol 24, , no. 3, pp. 261-284, Mar. 2003, doi: 10.1023/A:1021903132609.

[5] L. Kevin, W. R. Deal, V. Radisic, X. B. Mei, J.Uyeda, L. Samoska, A. Fung, T. Gaier, and R. Lai, "A 340-380 GHz integrated CB-CPW-towaveguide transition for sub millimeter-wave MMIC packaging," IEEE Microw. Wireless Compon. Lett, vol 19, no. 6, pp. 413-415, Jun. 2009, doi: 10.1109/LMWC.2009.2020043.

[6] T. Boon-Kok, S. Withington, and G. Yassin, "A compact microstrip-fed planar dual-dipole antenna for broadband applications," IEEE Antennas and Wireless Propag. Lett., vol 15, pp. 593-596, Jul. 2016, doi: 10.1109/LAWP.2015.2462114.

[7] T. Boon-Kok., and G. Yassin, "Unilateral finline transition at $\mathrm{THz}$ frequencies," In Proc. 20th Int. Symp. on Space Terahertz Technol., Charlottesville, USA, 2009, pp. 263-266.

[8] T. Boon-Kok, G. Yassin, P. Grimes, J. Leech, K. Jacobs, and C. Groppi, "A $650 \mathrm{GHz}$ unilateral finline SIS mixer fed by a multiple flare-angle smooth-walled horn," IEEE Trans. on Terahertz Sci. and Technol., vol 2, , no. 1, pp. 40-49, Jun. 2012, doi: 10.1109/TTHZ.2011.2177736.

[9] K. C. Gupta, "Finlines," in Microstrip Lines and Slotlines, 2nd ed. London, United Kingdom: Artech House, 1996, ch. 6, sec. 6,1, pp. 341344.

[10] C.Lopez, V. Desmaris, D. Meledin, A. Pavolotsky, and V. Belitsky, "Broadband Waveguide-to-Substrate Transition Using a Unilateral Etched Finline Structure," in Proc. 30th Int. Symp. on Space Terahertz Technol., Gothenburg, Sweden, 2019, pp. 47-48.

[11] C. Schieblich, J.K. Piotrowski and J.H. Hinken, "Synthesis of optimum finline tapers using dispersion formulas for arbitrary slot widths and locations," IEEE Trans. on Microw. Theory and techn., vol 32, , no. 12, pp. 1638-1645, Dec. 1984, doi: 10.1109/TMTT.1984.1132904.

[12] C. North, G. Yassin, and P. Grimes, "Rigorous analysis and design of finline tapers for high performance millimetre and submillimetre detectors," in Proc. 17th Int. Symp. on Space Terahertz Technol., Lerma, France, 2006, pp. 284-287.

[13] H. Rashid, D. Meledin, V. Desmaris, A. Pavolotsky, and V. Belitsky, "Superconducting 4-8-GHz hybrid assembly for $2 \mathrm{SB}$ cryogenic $\mathrm{THz}$ receivers.," IEEE Microw. Wireless Compon. Lett, vol 4, no. 2, pp. 193200, Mar. 2009, doi: 10.1109/TTHZ.2013.2294761.

[14] H. Rashid, S. Krause, D. Meledin, V. Desmaris, A. Pavolotsky, and V. Belitsky, "Frequency multiplier based on distributed superconducting tunnel junctions: Theory, design, and characterization," IEEE Trans. on Terahertz Sci. and Technol., vol 6, , no. 5, pp. 724-736, Sep. 2016, doi: 10.1109/TTHZ.2016.2583201.

[15] J. W. Kooi, C. K. Walker \& J. Hesler. (2009). A Broad Bandwidth Suspended Membrane Waveguide to Thin Film Microstrip Transition," [Online].Available:http://www.apc.univparis7.fr/ piat/BSD/Articles_file s/antenne1.pdf

[16] T. Boon-Kok, "Design of Unilateral Finline SIS Mixer" in Development of Coherent Detector Technologies for Sub-millimetre Wave Astronomy Observations, $1 \mathrm{st}$ ed. Switzerland, Springer International Publishing,2015,ch. 4, sec 4.2.1, pp 61.

[17] T. Boon-Kok., and G. Yassin, "A Slotline DC Block for Microwave, Millimeter, and Submillimeter Circuits," IEEE Microw. Wireless Compon. Lett., vol 29, no. 9, pp. 583-585, Sep. 2019, doi: 10.1109/LMWC.2019.2931467.

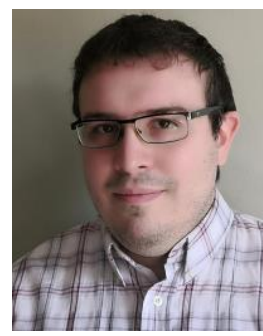

Cristian Lopez was born in Buenos Aires, Argentina, in 1990. He received the B.S. degree in electronic engineering from Facultad de Ingeniería del Ejército Grl. Div. Manuel N. Savio, Buenos Aires, Argentina, in 2012, and the M.Sc. degree in microelectronics from Universidad Politecnica de Cataluña, Barcelona, Spain, in 2018.

$\mathrm{He}$ is currently working toward the Ph.D. degree at Chalmers University of Technology, Gothenburg, Sweeden.
His current research interests are the design and characterization of cryogenic components for $\mathrm{THz}$ systems.

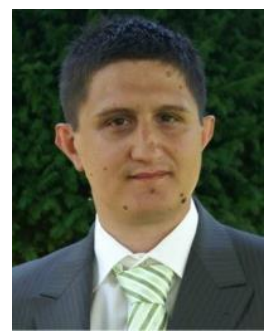

Vincent Desmaris received the M.Sc. degree in material science from the National Institute of Applied Science, Lyon, France, in 1999, and the Ph.D. degree in electrical engineering from the Chalmers University of Technology, Gothenburg, Sweden, in 2006. His thesis concerned the fabrication, characterization, and modeling of AlGaN/GaN microwave transistors. Since 2006, he has been with the Group for Advanced Receiver Development, Chalmers University of Technology. His research interests include the area of terahertz receiver technology, especially microfabrication and characterization of waveguide components and circuits, and planar cryogenic microwave devices.

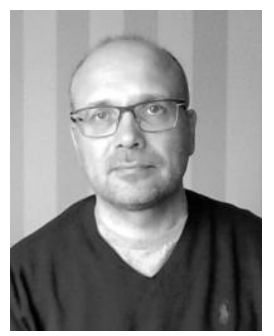

Denis Meledin received Ph.D. degree in radiophysics from MSPU, Moscow, Russia, 2003. From 2000 to 2003, he was a predoctoral fellow with Submillimeter Receiver Lab at Smithsonian Astrophysical Observatory, Cambridge, USA. Since 2003 he has been with Group for Advanced Receiver Development (GARD), Chalmers University of Technology, Gothenburg, Sweden. His work is related to development of instruments for radio-telescopes (e.g. for ALMA, APEX ). He focus on design, development and characterization of components for radioastronomy receivers operating at microwave and $\mathrm{mm} / \mathrm{submm}$ wavelengths. Beside that, he is involved in teaching of B.S and M.S. courses, and has been a co-supervisor of a number of Ph.D. students.

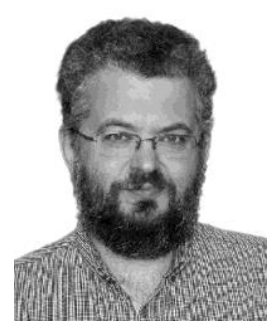

Alexey B. Pavolotsky received the M.S. and Ph.D. degrees from Moscow Aircraft Technology Institute / Technical University, in 1990 and 2003, both in material science and engineering.

Since 2002, he is with GARD, Group for Advanced Receiver Development, Onsala Space Observatory, Chalmers University of Technology, Gothenburg, Sweden. He currently holds a Senior Researcher position. His research interests include low-Tc superconducting thin film processing and characterization, as well as microfabrication in general. 


\section{ID T-TST-SPI-05-2020-00094.R1}

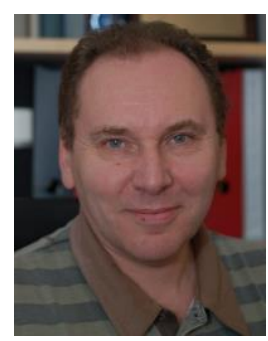

Victor Belitsky (M'95-SM'07) received the M.Sc. degree in electrical engineering from the Moscow Telecommunication Institute, Moscow, Russia, in 1977, and the $\mathrm{Ph} . D$. degree in experimental physics from the Institute of Radio Engineering and Electronics, U.S.S.R. Academy of Sciences, Moscow, Russia, in 1990. He is currently a Professor and Head of the Group for Advanced Receiver Development, Department of Space, Earth, and Environmental Sciences, Chalmers University of Technology, Gothenburg, Sweden. His research interests include terahertz and superconducting electronics and components, instrumentation for radio astronomy, and environmental science. 